\title{
Measuring cotinine to monitor tobacco use and smoking cessation
}

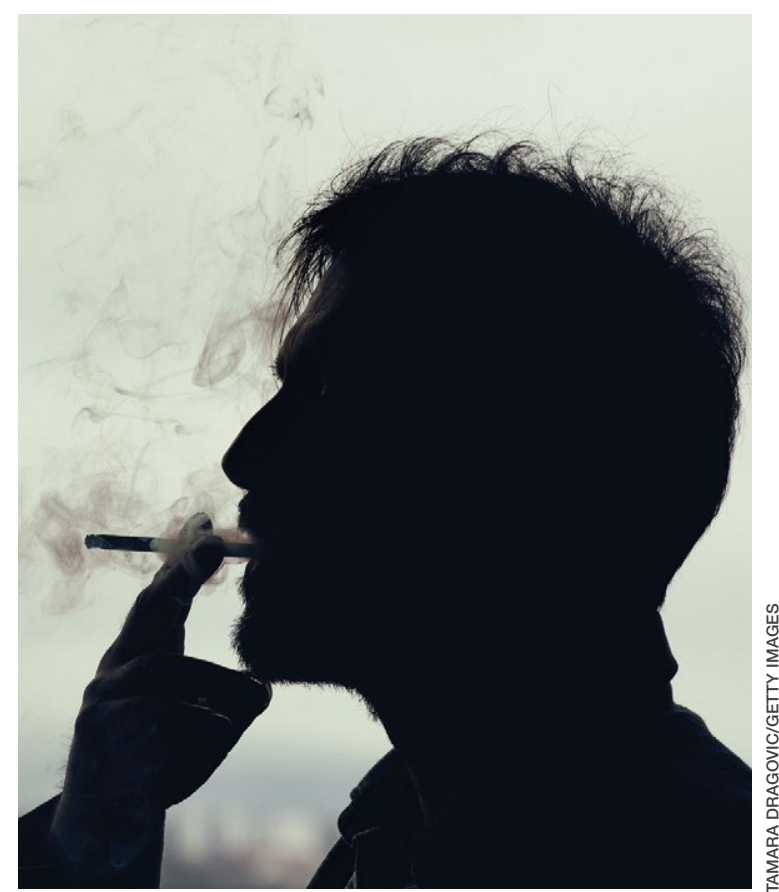

\section{Kimberly R. Stubbs, MD}

Department of Psychiatry and Behavioral Sciences Emory University School of Medicine Atlanta, Georgia

\section{Christopher R. Smith, MD}

Department of Psychiatry and Behavioral Sciences Emory University School of Medicine Atlanta, Georgia

\section{Yi-lang Tang, MD, PhD}

Department of Psychiatry and Behavioral Sciences Emory University School of Medicine

Atlanta, Georgia

Mental Health Service Line

Atlanta VA Medical Center

Decatur, Georgia

\section{For certain patients, cotinine screening may be a valuable addition to their treatment}

C igarette smoking is common among patients with schizophrenia, mood disorders, anxiety disorders, ${ }^{1-3}$ substance use disorders (SUDs), ${ }^{4}$ and other psychiatric disorders. Research suggests that compared with the general population, patients with SUDs consume more nicotine products and are more vulnerable to the effects of smoking. ${ }^{5}$ Despite the availability of effective treatments, many mental health professionals are reluctant to identify and treat tobacco use disorder, ${ }^{6-8}$ or they prioritize other disorders over tobacco use. Early detection and treatment of tobacco use disorder can improve patients' health and reduce the incidence of acute and chronic illness.

Cotinine is a biomarker that can be used to detect tobacco use. It can be measured in routine clinical practice by collecting urinary, serum, or salivary specimens, and used to monitor psychiatric patients' tobacco use. Monitoring cotinine levels is similar to using other biomarkers to assess medication adherence or identify illicit substance use. A growing body of evidence supports the utility of cotinine screening as a part of a comprehensive substance use disorder treatment plan, ${ }^{5,9,10}$ especially for:

- patients who have comorbid conditions that can be exacerbated by tobacco use, such as chronic obstructive pulmonary disease

- patients who are pregnant $t^{11,12}$

- patients who are less reliable in self-report or who require objective testing for validation.

\section{Disclosures}

The authors report no financial relationships with any companies whose products are mentioned in this article, or with manufacturers of competing products.

doi: 10.12788/cp.0115 


\section{Cotinine concentrations and cut-off levels}

aily smokers typically have a serum/plasma cotinine concentration of $\geq 100 \mathrm{ng} / \mathrm{mL}$. Individuals with heavy exposure to secondhand smoking may have plasma cotinine concentrations up to $25 \mathrm{ng} / \mathrm{mL}$, and urine samples tend to be much more specific. ${ }^{16}$ However, serum cotinine has a wide cut-off range due to diverse racial/ethnic, gender, and pregnancy-related variations; the wide range is also associated with genetic polymorphisms of cytochrome P450 2A6 alleles and nicotine's numerous metabolic pathways. ${ }^{11,18}$

Traditionally a serum/plasma cut-off point of approximately $15 \mathrm{ng} / \mathrm{mL}$ has been accepted to detect current tobacco use; however, recent studies ${ }^{21}$ recommend an average optimal cut-off point for US adults of $3 \mathrm{ng} / \mathrm{mL}$. This possibly reflects differences in national cigarette smoking patterns and exposure. ${ }^{21}$ One study suggested optimal cut-off differences for men $(1.78 \mathrm{ng} / \mathrm{mL})$ and women $(4.47 \mathrm{ng} / \mathrm{mL}){ }^{19}$ The same study also suggested different optimal cut-off levels for non-Hispanic White men $(6.79 \mathrm{ng} /$ $\mathrm{mL})$, non-Hispanic Black men (13.3 $\mathrm{ng} / \mathrm{mL})$, and Mexican-American men $(0.79 \mathrm{ng} / \mathrm{mL})$. $^{19}$ These researchers also suggested different optimal cut-off levels for non-Hispanic White women (4.73 ng/mL), non-Hispanic Black women $(5.91 \mathrm{ng} / \mathrm{mL})$, and Mexican-American women $(0.84 \mathrm{ng} / \mathrm{mL}) .{ }^{19}$ Genetic factors may also play a role in the progression of nicotine dependence and pose challenges that impact smoking persistence. ${ }^{20}$
Routine clinical screening of tobacco use is recommended for all patients and early detection may facilitate earlier treatment. Several FDA-approved medications are available for smoking cessation ${ }^{13}$; however, discussion of treatment options is beyond the scope of this review. In this article, we describe how cotinine is measured and analyzed, 3 case vignettes that illustrate its potential clinical utility, and limitations to its use as a biomarker of tobacco use.

\section{Methods of measuring cotinine}

Cigarette smoking is associated with the absorption of nicotine, which is mainly metabolized by cytochrome P450 (CYP) 2A6 to 6 primary metabolites: cotinine, hydroxycotinine, norcotinine, nornicotine, cotinine oxide, and nicotine oxide. ${ }^{14,15}$ Cotinine is the biomarker of choice for detecting use of tobacco/nicotine products due to its stability (it is not influenced by dietary or environmental factors), extended half-life (16 to 19 hours, compared with 2 hours for nicotine), and stable concentration throughout the day. Samples from saliva, urine, or blood can be analyzed through radioimmunoassay, enzyme-linked immunosorbent assay (ELISA), and gas/liquid chromatography. ${ }^{16}$ The specificity of cotinine for tobacco use is excellent, except for persons who are taking medications that contain nicotine. ${ }^{17}$
An advantage of cotinine over other biomarkers for smoking (such as carbon monoxide in expired air) is that the optimal cut-off points for cotinine are relatively uninfluenced by the prevalence of smoking in the population. The optimal cut-off levels used to detect current tobacco use may vary based on the sample or test used (saliva, urine, or plasma) and certain patient-specific factors (Box $\mathbf{1}^{11,16,18-21}$ ). However, for plasma or saliva cotinine, $16 \mathrm{ng} / \mathrm{mL}$ is the generally accepted cutoff level for detecting current tobacco use. A urinary cotinine cut-off level of $50 \mathrm{ng} / \mathrm{mL}$ is likely appropriate for most circumstances. ${ }^{17}$ Users of electronic nicotine delivery systems (electronic cigarettes) have been found to have cotinine levels similar to those of cigarette smokers. ${ }^{22}$

Assessment of cotinine levels in saliva may be considered for outpatient monitoring due to its noninvasive nature, tolerability, and the ability to collect multiple samples over a limited period. ${ }^{23}$ Saliva cotinine levels correlate closely with blood concentrations. Urine cotinine levels offer some advantage because concentrations are 6 times higher in urine than in blood or saliva. For this reason, urine cotinine is the most widely used biomarker in individuals who use tobacco due to its high sensitivity, specificity, reliability, and noninvasive collection. ${ }^{23}$ By using a lower urinary cut-off of $\geq 2.47 \mathrm{ng} /$ $\mathrm{mL}$, ELISA kits detect the highest sensitivity

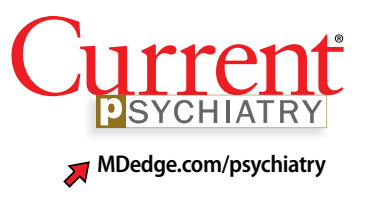

Clinical Point

Due to its stability, cotinine is the biomarker of choice for detecting use of tobacco/nicotine products

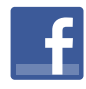

Discuss this article at www.facebook.com/ MDedgePsychiatry $\mathbf{Q}$ 


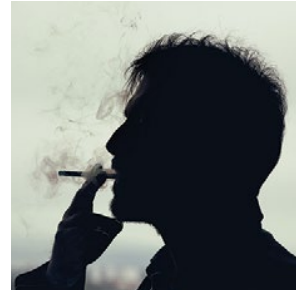

Monitoring tobacco use

\section{Clinical Point}

Routinely screening patients for smoking can be helpful due to potential interactions between tobacco smoke and psychotropics and specificity, which is useful for monitoring daily tobacco use. ${ }^{24}$ This cut-off value was associated with $100 \%$ sensitivity and specificity, and these numbers declined with increases in the cut-off threshold. ${ }^{23}$

\section{Impact of cigarette smoking}

The following 3 clinical vignettes illustrate the impact of tobacco use disorder on patients, and how cotinine might help with their treatment.

\section{Vignette 1}

Mr. D, age 44 , has a history of schizophrenia and has smoked 1 pack of cigarettes per day for the last 15 years. He was recently discharged from an inpatient psychiatric facility after his symptoms were stabilized. During his hospitalization, Mr. D used a nicotinereplacement product to comply with the hospital's smoke-free policy. Unfortunately, since discharge, Mr. D reports worsening auditory hallucinations despite adherence with his antipsychotic medication, clozapine, $600 \mathrm{mg}$ at bedtime. Collateral information gathered from Mr. D's mother confirms that he has been adherent with the discharge medication regimen; however, Mr. D has resumed smoking 1 pack of cigarettes daily. The treatment team suspects that his worsening psychosis is related to the decrease of blood clozapine level due to CYP induction by cigarette smoke.

\section{Cotinine and smoking-related drug interactions}

Vignette 1 illustrates the significant impact tobacco smoke can have on the effectiveness of a psychotropic medication. This is caused by polycyclic aromatic hydrocarbons induction of hepatic CYP1A2 isoenzymes. Clinicians should routinely screen patients for smoking status due to the potential for drug interactions. Common major CYP1A2 substrates include clozapine, olanzapine, duloxetine, and mirtazapine. Common minor CYP1A2 substrates include asenapine, chlorpromazine, haloperidol, perphenazine, ziprasidone, and zolpidem..$^{25}$ This case also highlights the potential advantage of collecting collateral information, and the utility of objective laboratory testing (such as cotinine monitoring) for optimal treatment because patient self-report can be unreliable and incomplete. ${ }^{12,26}$

\section{Vignette 2}

Mr. B, age 34 , has a history of cocaine use disorder and tobacco use disorder. He is referred to a treatment program and participates in a contingency management program for his substance use disorders. Biomarkers, including salivary cotinine, are used to assess Mr. B's exposure to tobacco use. Mr. B and other participants in his program are eligible for prize draws if they are found to have samples that are negative for tobacco and other substances. There are other incentives in place for patients who show a reduced cotinine concentration.

\section{Cotinine monitoring and contingency management}

Clinicians can incorporate cotinine monitoring into existing SUD treatment. This is similar to the utilization of other biomarkers that are commonly used to identify recent illicit substance use or monitor adherence to treatment medications. For example, benzoylecgonine, a metabolite of cocaine, is frequently used to monitor abstinence from cocaine. ${ }^{27}$ In addition, buprenorphine metabolites are used to monitor medication adherence and the possibility of diversion among patients being treated for opioid use disorder. ${ }^{28}$ Smoking cessation may be associated with a decreased risk of relapse to illicit substances. ${ }^{29}$

Treatments based on contingency management principles involve giving patients tangible rewards to reinforce desired (positive) behaviors. Smoking cessation can be confirmed by monitoring cotinine levels. Gayman et $\mathrm{al}^{9}$ found twice-weekly salivary testing was compatible with monitoring and promoting abstinence in a prize-based contingency management smoking cessation program. Most prior studies used urine cotinine measures to verify abstinence. Although highly reliable, urine samples require close monitoring to ensure sample validity, which can be a burden on staff and unpleasant for patients. ${ }^{9}$ It is also important to note that the rate of elimination of cotinine from saliva and urine are comparable. The half-life of cotinine is approximately 18 hours, and therefore the specificity of 


\section{Limitations of cotinine monitoring}

hallenges in the collection of samples, storage, shipping, and instrumentation may limit cotinine consistency as a dependable biomarker in the clinical setting..$^{23}$ Overall, quantitative measurements of cotinine have relative constructive utility in separating smokers from nonsmokers, because daily smokers typically have serum concentrations of $100 \mathrm{ng} / \mathrm{mL}$ or higher, in contrast to light/ non-daily smokers, who have cotinine concentrations $<10 \mathrm{ng} / \mathrm{mL}$. Even heavy exposure to secondhand smoke typically yields plasma concentrations up to approximately $25 \mathrm{ng} / \mathrm{mL}$. However, cotinine is a general metabolite found with the use of all nicotine products, which makes it extremely difficult to differentiate tobacco use from the use of nicotine replacement products, which are frequently used to treat tobacco use disorders.

One potential solution is to measure nicotine-derived nitrosamine ketone (NNK) and its metabolite 4-(methylnitrosamino)1-(3-pyridyl)-1-butanol (NNAL). Both NNK and NNAL are tobacco-specific lung carcinogens. NNAL can be measured in the urine. Although total NNAL represents only $15 \%$ of NNK dose intake, it has been quantified, with urine concentrations of $\geq 1,000 \mathrm{fmol} / \mathrm{mL}$ for daily smokers. NNAL also has an extremely high specificity to tobacco smoke, and thus allows differentiation of tobacco use from nicotine replacement treatment. Unfortunately, measurement for this biomarker requires specific chemical expertise and expensive equipment.

Another potential barrier to using cotinine in the clinical setting is the variable cut-off levels used in the United States, based on differences in race/ethnicity. This may be secondary to differences in smoking behaviors and/or differences in cotinine metabolism. ${ }^{21}$ salivary test strips may be impacted during the first 4 to 5 days of abstinence. In the first few days of smoking cessation, a more intensive approach, such as quantifying urine cotinine levels and monitoring decline, may be appropriate. ${ }^{23}$

\section{Vignette 3}

Ms. C, age 34 and pregnant, is admitted to an outpatient treatment program for alcohol use disorder. She also has generalized anxiety disorder and tobacco use disorder. In addition to attending group therapy sessions and self-reporting any recent alcohol consumption, Ms. C also undergoes alcohol breathalyzer tests and urine studies of alcohol metabolites to monitor abstinence from alcohol. She says that the regular laboratory screening for alcohol use gives her a sense of accountability and tangible evidence of change that positively impacts her treatment. When the treating psychiatrist recommends that Ms. C also consider addressing her tobacco use disorder, she asks if there is some way to include laboratory testing to monitor her smoking cessation.

\section{Cotinine as a predictor of smoking status}

Smoking abstinence rates during pregnancy are lower than that for other substances, and pregnant women may not be aware of the impact of smoking on fetal development. ${ }^{30}$ Cotinine can be used to verify self-report of smoking status and severity. ${ }^{10,31,32}$

Salivary cotinine tests are commercially available, relatively economical, and convenient to use when frequent monitoring is required..$^{32}$ In general, based on established cut-off values that are unique to the specimen collected, the overall high specificity and sensitivity of salivary testing allows clinicians to predict smoker vs nonsmoker status with confidence. For example, a 2008 study reported a salivary cotinine cut-off level of $12 \mathrm{ng} / \mathrm{mL}$ for smokers. ${ }^{21}$ The sensitivity and specificity of this cut-off value for distinguishing cigarette smokers from never smokers were $96.7 \%$ and $96.9 \%$, respectively. ${ }^{21}$

Additionally, some studies suggest that cotinine levels may be predictive of treatment outcomes and retention in SUD treatment programs. ${ }^{33,34}$ One study of smoking cessation using nicotine replacement products found that compared with patients with lower baseline cotinine levels prior to treatment, patients with higher baseline cotinine plasma levels had lower smoking cessation success rates. ${ }^{34}$

\section{Clinical Point}

Cotinine can be used to verify self-report of smoking status and severity 


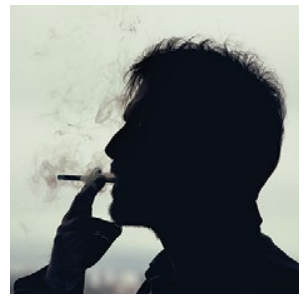

Monitoring tobacco use

\section{Clinical Point}

\section{Cotinine is not a} routine test and there are no guidelines on how or when it should be used

\section{Related Resources}

- Peckham E, Brabyn S, Cook L, et al. Smoking cessation in severe mental ill health: what works? An updated systematic review and meta-analysis. BMC Psychiatry. 2017; 17(1):252.

- Tidey JW, Miller ME. Smoking cessation and reduction in people with chronic mental illness. BMJ. 2015;351:h4065. doi: 10.1136/bmj.h4065

Drug Brand Names

Asenapine · Saphris Buprenorphine - Sublocade

Clozapine - Clozaril

Mirtazapine $\cdot$ Remeron

Olanzapine - Zyprexa

Duloxetine $\cdot$ Cymbalta

Ziprasidone. Geodon

Haloperidol • Haldol

\section{A few caveats}

There are several limitations to quantitative measures of cotinine (Box 2,1,23 page 17). These include (but are not limited to) potential errors related to sample collection, storage, shipping, and analysis. ${ }^{23}$ Compared with other methods, point-ofcare cotinine measurement in saliva is noninvasive, simple, and requires less training to properly use. ${ }^{23}$

Confirmation of smoking cessation can be monitored reliably within the clinical setting using cotinine monitoring. However, this is not a routine test, and there are no guidelines or consensus on how or when it should be used. The clinical feasibility of cotinine monitoring for psychiatric patients will depend on the cost of testing, methods used, amount of reimbursement for performing the tests, and how clinicians value such testing. ${ }^{35}$

\section{References}

1. Prochaska JJ, Das S, Young-Wolff KC. Smoking, mental illness, and public health. Annu Rev Public Health. 2017:38:165-185

2. Pal A, Balhara YP. A review of impact of tobacco use on patients with co-occurring psychiatric disorders. Tob Use Insights. 2016;9:7-12
3. Lawrence D, Mitrou F, Zubrick SR. Smoking and mental illness: results from population surveys in Australia and the United States. BMC Public Health. 2009;9:285.

4. Kalman D, Morissette SB, George TP. Co-morbidity of smoking in patients with psychiatric and substance use disorders. Am J Addict. 2005;14(2):106-123.

5. Baca CT, Yahne CE. Smoking cessation during substance abuse treatment: what you need to know. J Subst Abuse Treat. 2009;36(2):205-219.

6. Hall SM, Tsoh JY, Prochaska JJ, et al. Treatment for cigarette smoking among depressed mental health outpatients: a randomized clinical trial. Am J Public Health. 2006; 96(10):1808-1814.

7. McHugh RK, Votaw VR, Fulciniti F, et al. Perceived barriers to smoking cessation among adults with substance use disorders. J Subst Abuse Treat. 2017;74:48-53.

8. Strong DR, Uebelacker L, Fokas K, et al. Utilization of evidence-based smoking cessation treatments by psychiatric inpatient smokers with depression. J Addict Med. 2014;8(2): 77-83.

9. Gayman C, Anderson K, Pietras C. Saliva cotinine as a measure of smoking abstinence in contingency management - a feasibility study. The Psychological Record. 2017;67(2):261-272

10. Schepis TS, Duhig AM, Liss T, et al. Contingency management for smoking cessation: enhancing feasibility through use of immunoassay test strips measuring cotinine. Nicotine Tob Res. 2008;10(9):1495-1501.

11. Stragierowicz J, Mikolajewska K, Zawadzka-Stolarz M et al. Estimation of cutoff values of cotinine in urine and saliva for pregnant women in Poland. Biomed Res Int. 2013;2013:386784. doi.org/10.1155/2013/386784

12. Shipton D, Tappin DM, Vadiveloo T, et al. Reliability of sel reported smoking status by pregnant women for estimating smoking prevalence: a retrospective, cross sectional study. BMJ. 2009;339:b4347. doi.org/10.1136/bmj.b4347

13. Aubin HJ, Karila L, Reynaud M. Pharmacotherapy for smoking cessation: present and future. Curr Pharm Des. 2011;17(14):1343-1350.

14. McGuffey JE, Wei B, Bernert JT, et al. Validation of a LC-MS/ MS method for quantifying urinary nicotine, six nicotine metabolites and the minor tobacco alkaloids--anatabine and anabasine--in smokers' urine. PLoS One. 2014;9(7):e101816. doi: 10.1371/journal.pone.0101816

15. Duque A, Martinez PJ, Giraldo A, et al. Accuracy of cotinine serum test to detect the smoking habit and its association with periodontal disease in a multicenter study. Med Ora Patol Oral Cir Bucal. 2017;22(4):e425-e431. doi: 10.4317/ medoral.21292

16. Avila-Tang E, Elf JL, Cummings KM, et al. Assessing secondhand smoke exposure with reported measures. Tob Control. 2013;22(3):156-163

17. Benowitz NL, Bernert JT, Foulds J, et al. Biochemical verification of tobacco use and abstinence: 2019 Update Nicotine Tob Res. 2020;22(7):1086-1097.

18. Nakajima M TY. Interindividual variability in nicotine metabolism: c-oxidation and glucuronidation. Drug Metab Pharmaokinet. 2005;20(4):227-235

19. Benowitz NL, Bernert JT, Caraballo RS, et al. Optimal serum cotinine levels for distinguishing cigarette smokers and nonsmokers within different racial/ethnic groups in the United States between 1999 and 2004. Am J Epidemiol. 2009;169(2):236-248

continued on page 49

\section{Bottom Line}

Cotinine is a biomarker that can be used to detect tobacco use. Cotinine measurement can be used to monitor tobacco use and smoking cessation in psychiatric patients. Early detection and treatment of tobacco use disorder can improve patients' health and reduce the incidence of acute and chronic illnesses. However, cotinine measurement is not a routine test, and there are no guidelines 
20. Schnoll R, Johnson TA, Lerman C. Genetics and smoking behavior. Curr Psychiatry Rep. 2007;9(5):349-357.

21. Kim S. Overview of cotinine cutoff values for smoking status classification. Int J Environ Res Public Health. 2016;13(12):1236.

22. Etter JF, Bullen C. Saliva cotinine levels in users of electronic cigarettes. Eur Respir J. 2011;38(5):1219-1220.

23. Raja M, Garg A, Yadav P, et al. Diagnostic methods for detection of cotinine level in tobacco users: a review. J Clin Diagn Res. 2016;10(3):ZE04-06. doi: 10.7860/ JCDR/2016/17360.7423

24. Balhara YP, Jain R. A receiver operated curve-based evaluation of change in sensitivity and specificity of cotinine urinalysis for detecting active tobacco use. J Cancer Res Ther 2013;9(1):84-89.

25. Fankhauser M. Drug interactions with tobacco smoke: implications for patient care. Current Psychiatry. 2013;12(1):12-16.

26. Scheuermann TS, Richter KP, Rigotti NA, et al. Accuracy of self-reported smoking abstinence in clinical trials of hospital-initiated smoking interventions. Addiction. 2017;112(12):2227-2236

27. Holtyn AF, Knealing TW, Jarvis BP, et al. Monitoring cocaine use and abstinence among cocaine users for contingency management interventions. Psychol Rec. 2017;67(2):253-259.

28. Donroe JH, Holt SR, O'Connor PG, et al. Interpreting quantitative urine buprenorphine and norbuprenorphine levels in office-based clinical practice. Drug Alcohol Depend 2017;180:46-51.

29. Sullivan M, Covey, LS. Current perspectives on smoking cessation among substance abusers. Curr Psychiatry Rep. 2002;4(5):388-396.

30. Forray A, Merry B, Lin H, et al. Perinatal substance use: a prospective evaluation of abstinence and relapse. Drug Alcohol Depend. 2015;150:147-155.

31. Parker DR, Lasater TM, Windsor R, et al. The accuracy of self-reported smoking status assessed by cotinine test strips. Nicotine Tob Res. 2002;4(3):305-309.

32. Asha V, Dhanya M. Immunochromatographic assessment of salivary cotinine and its correlation with nicotine dependence in tobacco chewers. J Cancer Prev. 2015;20(2):159-163.

33. Hall S, Herning RI, Jones RT, et al. Blood cotinine levels as indicators of smoking treatment outcome. Clin Pharmacol Ther. 1984;35(6):810-814

34. Paoletti P, Fornai E, Maggiorelli F, et al. Importance of baseline cotinine plasma values in smoking cessation: results from a double-blind study with nicotine patch. Eur Respir J. 1996;9(4):643-651.

35. Montalto NJ, Wells WO. Validation of self-reported smoking status using saliva cotinine: a rapid semiquantitative dipstick method. Cancer Epidemiol Biomarkers Prev. 2007;16(9):1858-1862 